\title{
ARREGLO DE ESTELAS CALENDÁRICAS DE DZIBILCHALTÚN: CONOCIMIENTO GENOMÓNICO Y SU RELACIÓN CON EL PODER
}

\author{
Setting calendar stelles of dzibilchaltún: gnomonic knowledge \\ and its relationship with the power
}

EPISTEMUS

ISSN: 2007-8196 (electrónico)

ISSN: 2007-4530 (impresa)

\section{Dr. Raúl Pérez-Enríquez}

Recibido: 16 de agosto de 2016, Aceptado: 29 de junio de 2017

Autor de Correspondencia: Dr. Raúl Pérez Enríquez

Correo:raul.perez@unison.mx

\section{Resumen}

Un aspecto cultural que sugiere la existencia de un Poder, sea éste religioso o político, es la presencia de estructuras calendáricas. La obsesión por la medición del Tiempo y sus manifestaciones es una de las características de la Cultura Maya. Ejemplos como el Castillo en ChichenItzá y el Templo Redondo de Mayapán muestran el nivel de conocimiento del movimiento aparente del Sol alrededor de la Tierra que tenían los mayas. En este trabajo, se analiza desde el punto de vista gnomónico, el conjunto de Tres Estelas de Dzibilchaltún ubicadas al inicio del Sacbé 1. Su orientación, casi exacta Norte-Sur, refleja una intensión gnomónica (miran hacia el Sol del mediodía), y las alturas sugieren una intensión calendárica; esto es, debido a su ubicación (Latitud $21.092^{\circ}$ y orientación $187^{\circ}$ de azimut) la sombra de un gnomon en el Solsticio de Invierno es igual a su altura, mientras el 'factor gnomónico' correspondiente es igual a la unidad. El astrónomo-sacerdote conocía estas características y podía predecir las épocas del año.

Palabras clave: Tiempo, factor gnomónico, poder, rituales, solsticios.

\section{Abstract}

A cultural feature that suggests the existence of a power, whether religious or political, is the presence of calendrical structures. The obsession about measurement of Time and its manifestations is well known in the Maya culture. Examples like El Castillo in Chichenitza and the Templo Redondo of Mayapán show the kind of knowledge the Maya had about the apparent motion of the Sun around the Earth. In this work, the set of three steles of Dzibilchaltún, located at the beginning of the Sacbé 1, is analysed from the gnomonic point of view. Its orientation, almost exact North-South, projects a gnomonic purpose (they look towards the Sun of the midday), and the heights suggest a calendrical meaning; due to its location (latitude $21.092^{\circ}$ and $187^{\circ}$ azimuth) the shadow of a gnomon at Winter Solstice is equal to his height, while the corresponding 'gnomonic factor' is equal to the unit. The astronomer-priest knew these features and he was aware of seasons of the year.

Keywords: Time, gnomonic factor, power, rituals, solstices. 


\section{INTRODUCCIÓN}

De igual manera a como sucedió en otras latitudes, en Mesoamérica, los conocimientos astronómicos estuvieron íntimamente ligados a los ritos y mitos religiosos de los pueblos. A pesar de las distancias espaciales y temporales en las que se desarrollaron las diversas culturas de la región mesoamericana, ellas compartieron una cosmovisión central en la que el Tiempo, su conocimiento y su medición, fue central. La observación del cielo y sus manifestaciones era una manera de comunicarse y establecer contacto directo con los dioses [1].

Los rituales formaban parte de la estructura de poder en la que los gobernantes y sacerdotes-astrónomos, a través de ellos, podían perpetuar su presencia generación tras generación. En consecuencia, los rituales no pueden ser vistos como elementos que preservan o encarnan un conjunto de creencias religiosas, más bien deben verse como constructores, creadores o modificadores de las creencias religiosas [2]. Recordamos estas ideas acerca de los ritos o rituales porque en las Estelas Mayas se recogen escenas de ceremonias o eventos ligados a danzas u otro tipo de actividad de la élite [3], estrechamente ligados a los fechas calendáricas.

Así, las Estelas que son una de las expresiones de la escultura monumental Maya mejor conocidas y frecuentemente citadas, tuvieron su auge en el período Clásico (alrededor de 250 - 850 dC) [4]. Y existen vestigios y ejemplos de ellas en las más variadas formas y contenidos; sin embargo, se pueden también encontrar otras que no se encuentran talladas y grabadas. Estas estructuras, cuyo glifo 'Lakam-tun' fue descubierto desde el siglo pasado y que podría traducirse como "banner stone" o "Piedra Letrero", resultan por demás indicativas de actividades rituales. Su acepción como 'bandera' o 'letrero' es apropiada pues el glifo suele estar representado en esculturas como la Estela 11 de Yaxchilán [4].

Así, estelas y fechas calendáricas están estrechamente vinculadas a la estructura de poder. Sin embargo, solo la observación del cielo y el registro por décadas del paso de los días pudo permitir con la ayuda del sistema numérico vigesimal, la consolidación de un sistema calendárico. Para hacerlo, se requería no solo una organización social que permitiera la existencia de los sacerdotes-astrónomos

quienes llevarían las cuentas y la celebración de los rituales sino, también, de una élite gobernante que fusionada con ellos ejerciera el poder. Mismo que permitiría la organización de la gente para la erección de los templos y edificios, de los monumentos y de las estelas, en fin, de la producción de todos los materiales en que esos conocimientos se plasmarían.

En efecto, entre los Mayas, el astrónomo-sacerdote jugaba un papel

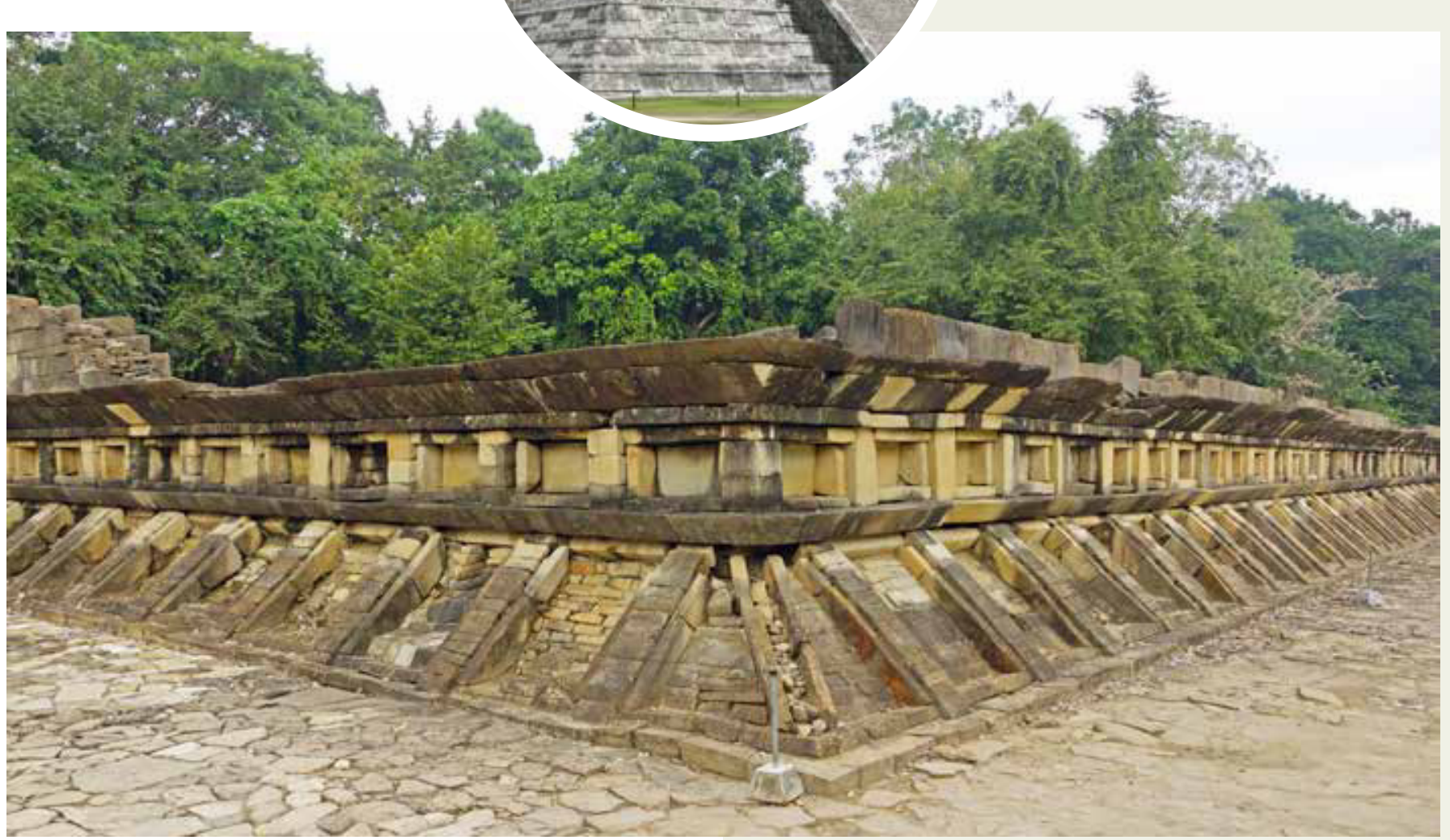



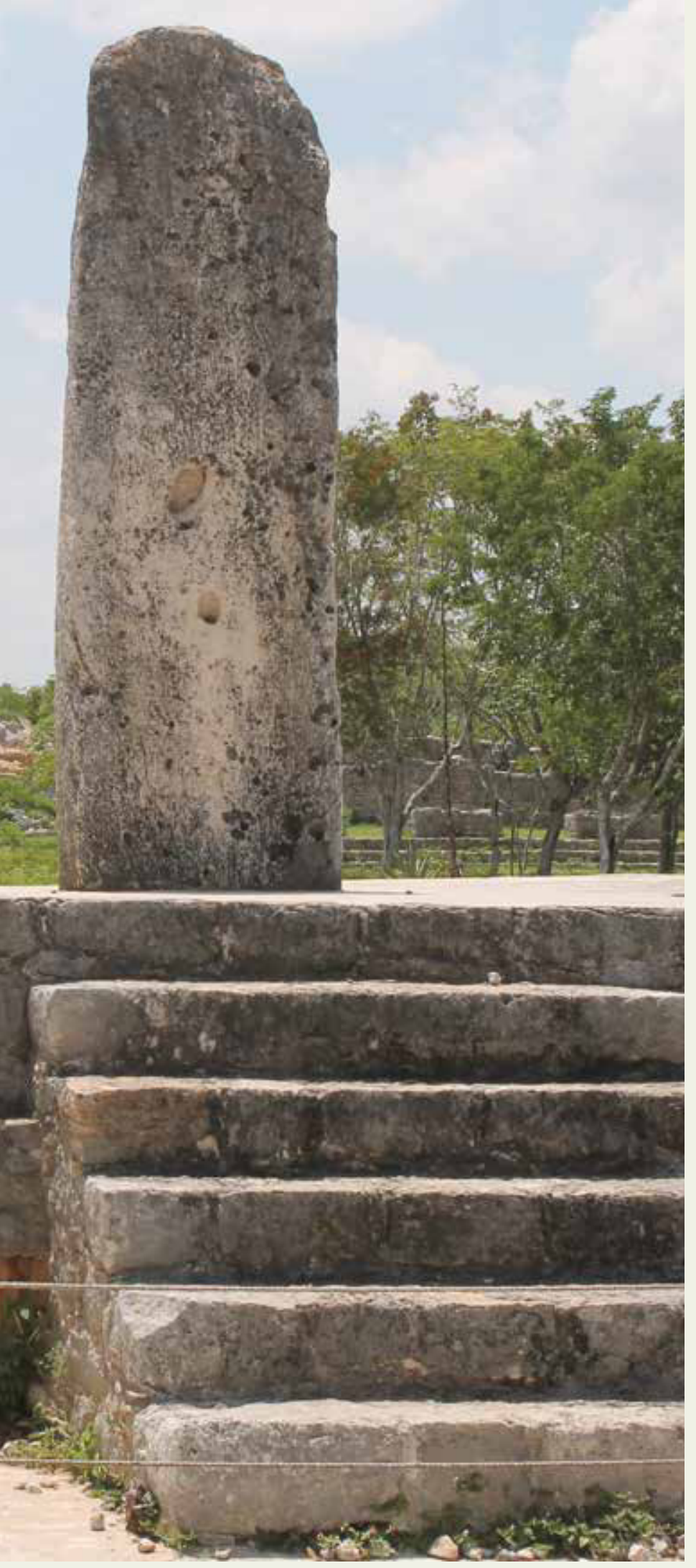

relevante de la estructura social; él era el ah miatz tu ximbal (el "sabio en el camino de las estrellas") [1], encargado de regular las actividades terrenas a partir de las acciones en el cielo. Entre dichos eventos que ocurren en el cielo, es imposible no considerar aquellos atribuidos al Sol, sean ellos durante sus ortos y ocasos, sean en los momentos de su culminación, al cruzar día con día la línea Meridiana.

Pero, como dice Renfrew, desafortunadamente, "El arqueólogo... no puede observar creencias: uno solo puede trabajar con vestigios, consecuencia de acciones. En los casos favorables... estos vestigios son el resultado de acciones cuya interpretación puede ser plausible como resultado 'de' una creencia religiosa" (citado por [2]). En otros casos, esto es imposible. Pero en general, se puede recurrir a instrumentos y simulaciones que puedan dar luz acerca de las acciones o motivos que le dieron origen.

El Calendario o, mejor aún, los Calendarios fueron unos de los productos mejor acabados de esa"relación del hombre con los dioses" a través del sacerdote-astrónomo. Los modelos elaborados para entender los ciclos existentes en la Naturaleza tuvieron, en Mesoamérica, una expresión superior en muchos aspectos a la de los conquistadores, al conjugar los ciclos meteorológicos y de los astros con otros creados matemáticamente. En la zona Maya, en particular, fue el tzolkin (ciclo de 260 días) y el haab (ciclo de 360 días y 5 días aciagos o "sin nombre"), amparados bajo la Cuenta Larga, lo que proporcionó uno de los sistemas Calendáricos más precisos de la antigüedad.

El Sistema Calendárico, los rituales ligados a él y la existencia de la élite gobernante son las expresiones más acabadas de la existencia de un Poder en la zona Maya de Mesoamérica. Es, a través del análisis de un vestigio concreto en Dzibilchaltún que ejemplificaremos esta relación: Las Tres Estelas alineadas S4, S5 y S6 ubicadas en las estructuras Str. 47, Str. 48 y Str. 49, respectivamente. En consecuencia, el objetivo del presente trabajo es el de proponer, a partir del análisis de algunas de las características de las Estelas S4, S5 y S6, ubicadas en sus plataformas, que estas estelas constituyeron un conjunto diseñado con objetivos gnomónicos en mente; en particular, el medir los solsticios y el paso zenital del Sol. Además, sugerir su uso como parte de un ritual en una fase de Dzibilchaltún en la que prácticamente no se construyeron nuevas edificaciones.

\section{DZIBILCHALTÚN, YUCATÁN}

Localizado a medio camino entre Mérida y Progreso, Dzibilchaltún aparece en el mapa como una pequeña ciudad que se desarrolló a lo largo de varios siglos. Con un nombre cuyo significado podría traducirse como "Donde hay escritos sobre piedras planas" [5], esta ciudad maya realmente representó un magnífico asentamiento de varias decenas de kilómetros cuadrados. Nosotros podríamos decir: "Lugar donde hay mensajes en las piedras planas"; mensajes cifrados en las estelas del sitio. 


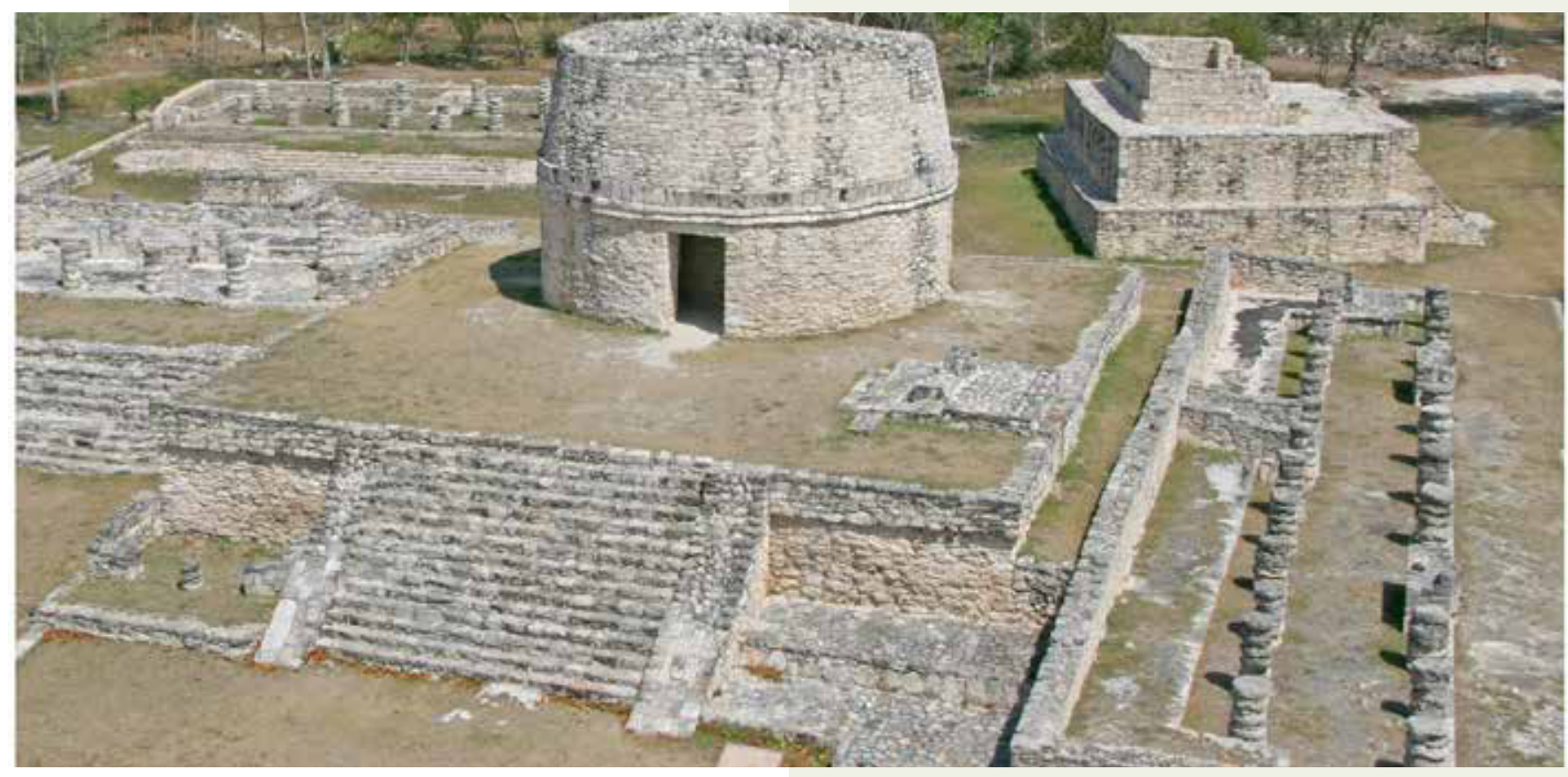

A pesar de la cercanía de esta ciudad con la costa (realmente está a un tercio de la distancia entre Mérida y Progreso), el lugar recibe muy poca agua de la lluvia. Por ello, también, los días despejados son más numerosos que en Progreso pero menos que en Mérida. La zona central del Sitio de alrededor de $500 \mathrm{~m} 2$, fue habitada por una elite de personajes importantes; y hubo, además, un desarrollo de pequeñas concentraciones de población satélites alrededor que se extendían en un área de 4 a 6 Km2 y más [6].

La población residente de Dzibilchaltún en el período Decadente estaba dispersa. La mayoría vivía en zonas alejadas del centro del sitio y, seguramente, en viviendas hechas de material perecedero. Una de las más claras evidencias de la escasa población la constituye la casi ausencia de entierros en ese período [7] y el hecho de que un pequeño porcentaje de los vestigios arqueológicos recuperados durante las excavaciones sean del período Floreciente Modificado o del Decadente propiamente dicho [6].

Las actividades rituales, principal orientación de la ciudad en este período, continuaron incluso una vez que la Conquista Española ya había llegado. Un testimonio de la vocación ritual del Dzibilchaltún lo puede constituir la Capilla abierta que levantaron los españoles con la finalidad, muy probablemente, de reorientar las actividades religiosas del sitio. Si bien, los desarrollos arquitectónicos del período Decadente que abarca de 1200 a 1540 dC, se orientaron a mejorar y remozar las estructuras que ya existían en los períodos anteriores, se pueden identificar estructuras específicas levantadas en ese período.

Muchas de ellas fueron parte de zonas habitacionales hechas de materiales perecederos; sin embargo, las Str. 47, Str. 48 y Str. 49, con sus estelas parecen haber sido diseñadas con un objetivo ritual [8]. Algunas de las obras relacionadas con actividades rituales que destacan en este período son, por ejemplo, el tunelamiento de la Str. 1 por su lado oeste para llegar y abrir espacio hacia la fachada de la estructura Str. 1-sub, para permitir el acceso a la cámara central; $y$, la construcción del altar con su vista hacia la antigua entrada al recinto [7]. Así, en este período Decadente, en el cual se consolidaron cambios estructurales importantes, se puede identificar ese enfoque puramente ritual de Dzibilchaltún con las tres pequeñas plataformas con estelas (recién mencionadas, Str. 47, Str. 48, Str. 49) que se describen en detalle más abajo, mismas que parecen haber sido planeadas en el marco de un plan integral. La conformación de las estelas sugiere que fueron talladas hacia el Período Temprano II. Lo que distingue a estas estelas es el que se encuentran ubicadas en una plataforma específicamente diseñada para cada una de ellas.

En Mayapán, otro sitio del norte de la Península de Yucatán, existen numerosas estelas pero solo unas cuantas están colocadas en plataformas construidas exprofeso. No cabe duda de que en Mesoamérica, la región más prolífera en el levantamiento de estelas es la región Maya. Como afirma Susan T. Evans, gracias al desciframiento de la lengua, se puede constatar que los integrantes de esta cultura se referían a ellas como "grandes rocas" o "rocas letrero" y explican los métodos usados para su erección [9].

Pero estas estelas se distinguen, también, porque las estructuras y ellas mismas están orientadas en la dirección Norte - Sur; aunque como se verá más adelante, es necesario precisar que su eje apuntaría a una dirección más precisa: azimut $187.6^{\circ}$ [10], como se puede observar en la Figura 1. 


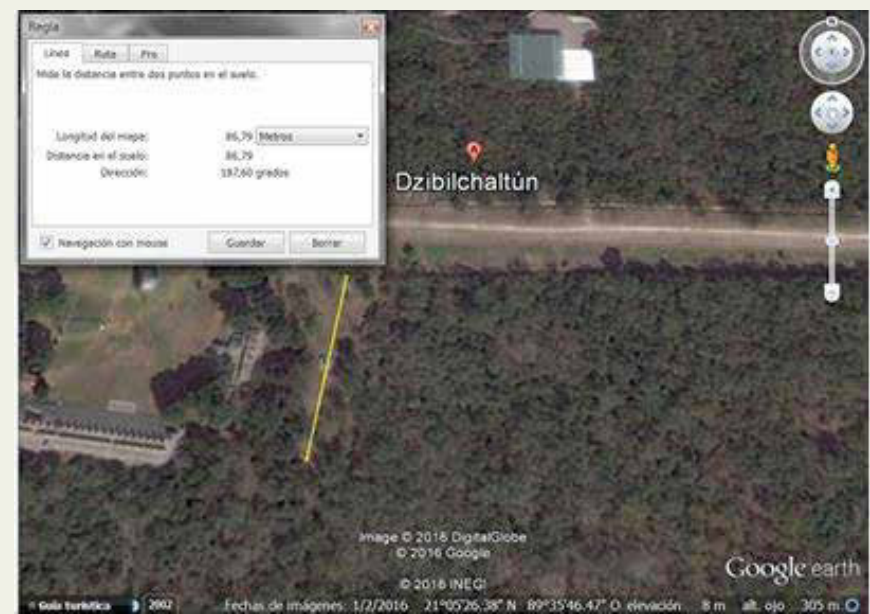

Figura 1. Foto de GoogleEarth en la que aparece la orientación del Arreglo de Estelas con azimut de $187.6^{\circ}$ (GoogleEarth, 2016).

No es descabellado pensar que el uso de las estelas pudo estar relacionado con la medición y registro del transcurrir del Tiempo. Si en otras regiones del mundo, Babilonia o Egipto, los movimientos del Sol fueron observados y registrados por medio del uso del gnomon entendido éste como una vara recta colocada verticalmente sobre una superficie horizontal [11], las estelas mayas bien pudieron jugar un papel similar en Mesoamérica. En donde, también podemos afirmar, hubo un extendido uso de bastones y báculos ceremoniales que en algún momento pudieron ser utilizados como gnómones. Postes, báculos y estelas pudieron ser utilizados en estas latitudes como dispositivos para registrar el Tiempo y bien pudieron haber evolucionado a partir de la estaca o vara de madera utilizada como herramienta para el cultivo [3].

\section{ARREGLO DE ESTELAS CALENDÁRICAS}

Al inicio del Sacbé1 que conduce hacia el Templo de las Muñecas se pueden observar tres estructuras alineadas en una dirección prácticamente Norte - Sur. Estas estructuras, denominadas Str. 47, Str. 48 y Str. 49, se encuentran alrededor de 20 metros hacia el Este de la estructura Str. 42 como se ilustra en el diagrama de la Figura 1. Al centro de cada una de las tres estructuras se encuentra colocada una estela (estelas S4, S5 y S6, respectivamente); mismas que han sido datadas para el período Decadente de Dzibilchaltún. Dos de las plataformas, Str. 48 y Str. 49, fueron estudiadas por Adrian D. Anderson (citado por [3]) y por los hallazgos hechos en el material de relleno de las estructuras y por la forma en la que los bloques de roca estaban unidos con mortero, una técnica no encontrada en estructuras más antiguas del sitio, le permitieron asignar una fecha dentro de ese período. La Estructura Str. 47 fue liberada hasta la temporada 2001-2002 del Proyecto Arqueológico Dzibilchaltún [12]; habiéndose encontrado muy pocos vestigios cerámicos o de otros materiales.

Las características de las estelas, en cuanto a formas y acabados, son diferentes a aquellas presentes en otras zonas de la cultura Maya. Se trata de estelas delgadas sin grabados aparentes y con su borde superior redondeado. Más bien, se supone que las caras rugosas de las estelas pudieron haber estado suavizadas con una capa de estuco, aunque, según Anderson (citado por [3)), al día de la excavación ya no se apreciaba resto alguno de él.

Si bien las tres estructuras son similares, ellas difieren en varios aspectos: la presencia de escalinatas y las alturas de las estelas colocadas en ellas. Respecto de las escalinatas, se puede afirmar, según se observa ahora después de

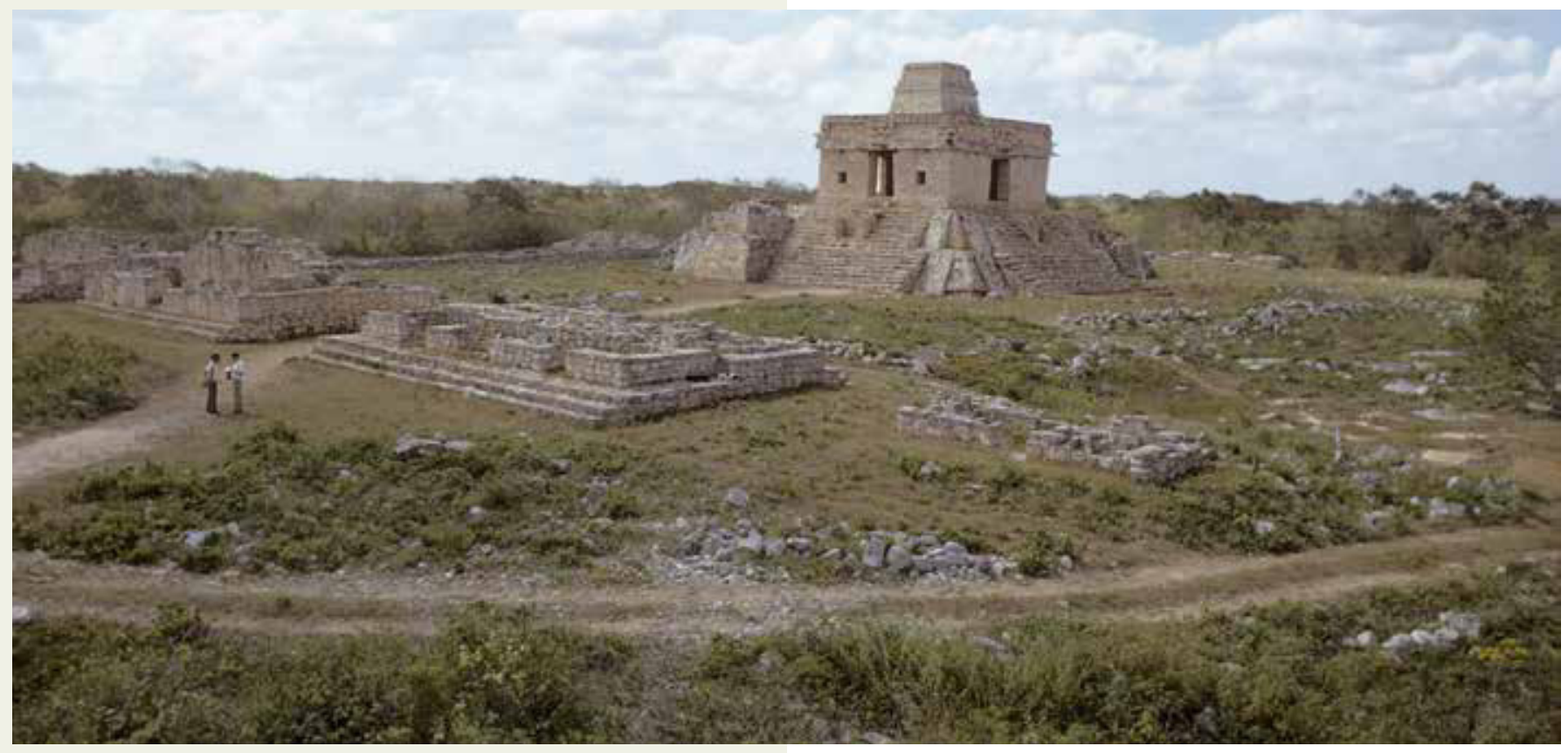


la recuperación arqueológica, que la Str. 48, ubicada entre las otras dos estructuras, posee dos: una hacia el Norte, mirando hacia la Str. 47; y, otra al Sur con vista hacia la Str. 49. Por su parte, las Str. 47 y Str. 49 , poseen una sola escalinata mirando cada una hacia la Str. 48; esto es, la Str. 47 con escalinata hacia el Sur, misma que fue propuesta por los arqueólogos [11] y la Str. 49 con la suya hacia el Norte, de cuya evidencia dan cuenta Andrews IV y Andrews V [6]. En la Figura 2, se puede apreciar el diagrama esquemático de este conjunto (las dimensiones no son a escala).
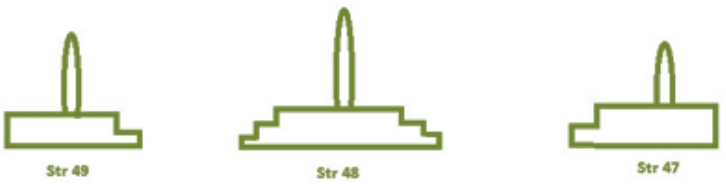

Figura 2. Esquemático del Arreglo de las Estelas en sus plataformas.

Ya mencionábamos arriba que el alineamiento de las plataformas y por ello de las estelas, es en la dirección Norte - Sur; aunque es importante para nuestra argumentación definir una orientación más precisa: azimut $187.6^{\circ}$; o bien, la sombra de un gnomon producida por el Sol del Solsticio de Invierno se observaría con dirección de $7.6^{\circ}$ al Este del Norte [10], como se puede observar en la Figura 1. Debemos destacar que dicha sombra debería ser igual

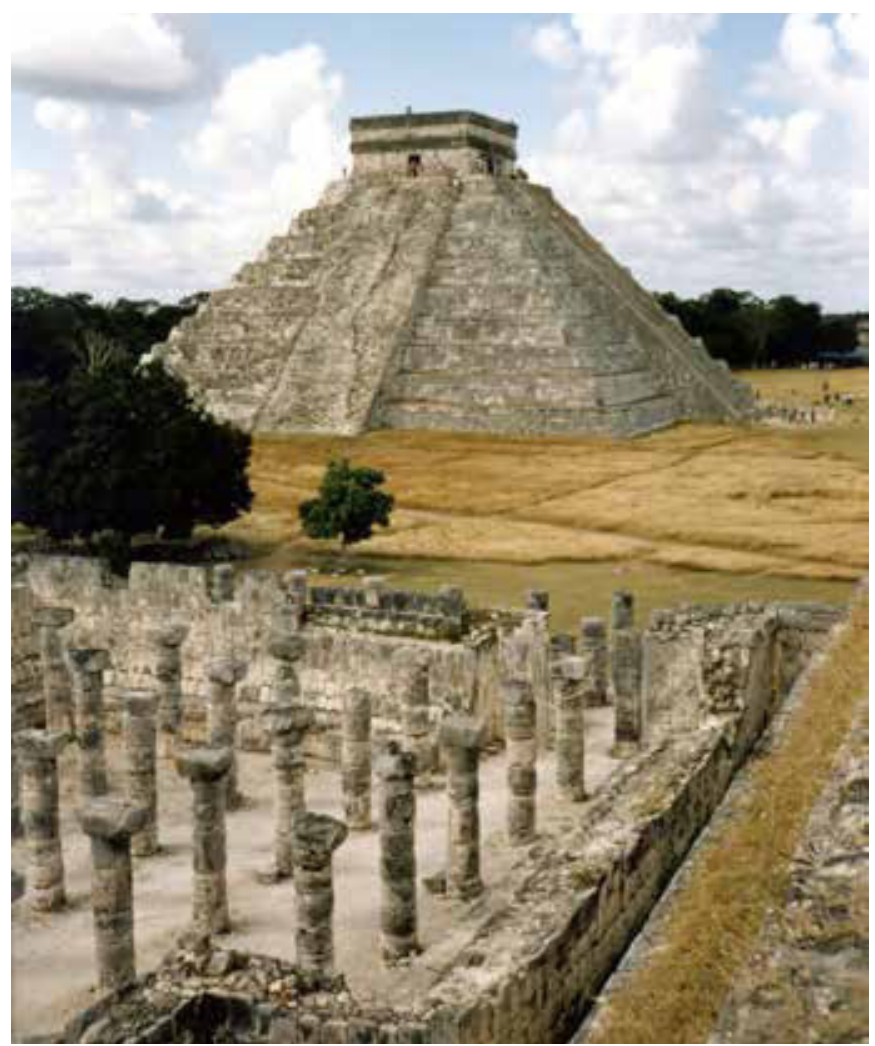

al gnomon pues la elevación del Sol sería, en aquel momento, de $45^{\circ}$.

Otro aspecto relevante es que las tres estructuras son casi rectangulares: Str. 48 con lados de 490 (oeste), 510 (este), 580 (norte) y $635 \mathrm{~cm}$ (sur); Str. 49 con lados de 540, 520,610 y $580 \mathrm{~cm}$, en el mismo orden [6); la Str. 47 quedó reconstruida de forma cuadrangular con lados 640 (eje este-oeste) por $690 \mathrm{~cm}$ (eje norte-sur) [12,13].

En la Tabla 1, se recogen las dimensiones básicas de las estelas tal y como quedaron reconstruidas en 2002. En ella, cabe destacar, las alturas de las mismas y la orientación general que no es en dirección exactamente nortesur sino ligeramente hacia el este del norte.

Tabla 1. Dimensiones de la Estelas.

\begin{tabular}{|l|c|c|c|}
\hline Concepto & \multicolumn{3}{|c|}{ Estelas } \\
\hline Largo $(\mathrm{cm})^{1}$ & S4 & S5 & S6 \\
\hline ancho $(\mathrm{cm})^{1}$ & 102 & 312 & 319 \\
\hline espesor $(\mathrm{cm})^{1}$ & 23 & 23 & 115 \\
\hline Altura $(\mathrm{cm})^{1}$ & 205 & 245 & 25 \\
\hline Orienta $\left({ }^{\circ}\right)^{2}$ & 187.6 & 187.6 & 187.6 \\
\hline Latitud $\left({ }^{\circ}\right)^{2}$ & 21.092 & 21.091 & 21.091 \\
\hline Longitud $\left({ }^{\circ}\right)^{2}$ & 89.596 & 89.596 & 89.596 \\
\hline
\end{tabular}

${ }^{1}$ Informe Técnico Temporada 2001-2002

(Maldonado et al) ${ }^{2}$ GoogleEarth (2016)

Deseamos destacar la importancia del mediodía para los Maya: Un día (parte diurna y nocturna) se contaba a partir del Sol de mediodía; cruce del Sol por la línea Meridiana, diríamos ahora. Este día recibía el nombre de uay. Ellos deben haber contado con métodos para determinar ese momento preciso que recibía el nombre de Zu huy kin (o mediodía en punto) [15); según Barrera Vásquez, los Maya podían hacer referencia a ese momento con la expresión Pochek' Bo'oy que literalmente significa "cuando el Sol hace sombra derecha" [15]. Esta sencilla expresión nos indica que ellos debieron utilizar algún elemento vertical (estela, poste, bastón, vara) cuya sombra pudieron observar para determinar cuándo ésta estaban derecha o recta; es decir, los mayas deben haber tenido el concepto de elemento que ahora llamamos gnomon como indicador.

Por ello, ese Sol de mediodía debió jugar un papel relevante en las ceremonias y rituales, tanto en las épocas de su apogeo como en el período Decadente. Habría ceremonias rituales diarias pero, sobre todo, de inicio y de fin del año. Se puede sugerir, asimismo, el papel relevante que jugaban los días de los Solsticios pues a los sitios en el horizonte de los ortos y ocasos correspondientes, los denominaban "las esquinas del Mundo" [16]. 
Así, combinando ambas acepciones podemos, ahora, valorar el momento de culminación del Sol en el Solsticio de Invierno como un momento crucial. Además, como se puede ver en la Tabla 2, en Dzibilchaltún, el Sol se encuentra exactamente entre el horizonte y el zenit; esto es, tiene una elevación de $45^{\circ}$. Esta posición implica un factor gnomónico platónico igual a cero $(\mathrm{fgp}=0)$ [17]. En cuanto al Solsticio de Verano, el Sol prácticamente se encuentra en el zenit: los mayas dirían Kaz chumuc kin [18].

Tabla 2. Alturas y Sombras de las Estelas.

\begin{tabular}{|l|c|c|c|}
\hline & S4 & S5 & S6 \\
\hline SI & 45.382 & 45.409 & 45.409 \\
\hline SI' & 45 & 45 & 45 \\
\hline SIs & 202.285 & 241.527 & 230.683 \\
\hline SIs' & 205.000 & 245.000 & 234.000 \\
\hline SV & 87.553 & 87.553 & 87.553 \\
\hline SV' & 87.529 & 87.529 & 87.529 \\
\hline SVs & 8.762 & 10.471 & 10.001 \\
\hline SVs' & 8.846 & 10.572 & 10.097 \\
\hline & & & \\
\hline SIs+SVs & 211.047 & 251.998 & 240.684 \\
\hline Sis'+SVs' & 213.846 & 255.572 & 244.097 \\
\hline SIs+SVs+e & 234.047 & 274.998 & 265.684 \\
\hline Sis'+SVs'+e & 236.846 & 278.572 & 269.097 \\
\hline & & & \\
\hline Fg $^{\prime}$ & 1.0295 & 1.0286 & 1.0286 \\
\hline fg' $^{\prime}$ & 1.0431 & 1.0431 & 1.0431 \\
\hline Fge $^{\prime}$ & 1.1417 & 1.1224 & 1.1354 \\
\hline fge' $^{\prime}$ & 1.1553 & 1.1370 & 1.1500 \\
\hline Fgp $^{\prime}$ & -0.0130 & -0.0140 & -0.0140 \\
\hline fgp' $^{\prime}$ & 0.0000 & 0.0000 & 0.0000 \\
\hline
\end{tabular}

$\mathrm{Sx}$ - Elevaciones del Sol en Solsticios, $\mathrm{x}=\mathrm{I}$ para Invierno y $\mathrm{x}$ $=\mathrm{V}$ para Verano

Sxs - Correspondientes valores de las sombras

fg - Factores gnomónicos

' - Las primadas corresponden a la orientación general de las estructuras.

Los días del paso del Sol por el zenit, fecha calendárica de suma importancia en la cultura Maya, pueden ser apreciados con la ayuda de las estelas. Las fechas correspondientes son los días 26 de mayo y 18 de julio y en esos días las estelas no proyectan sombra a mediodía.

La idea que propone la planeación del conjunto de estelas como un todo de objetivo ritual, se ve soportada cuando se observan los resultados de las mediciones de las sombras de las estelas y sus respectivas alturas. Por ejemplo, la longitud de las sombras de la estela 54 en los solsticios más el espesor de la misma $(234,047 \mathrm{~cm}$ en la Tabla 2) es igual a la altura de la estela $S 6(234.0 \mathrm{~cm})$; también, La suma de las sombras de la estela $\mathrm{S} 6$ en los solsticios $(244,097 \mathrm{~cm}$ en la Tabla 2) es igual a la altura de la estela S5 $(245,0 \mathrm{~cm})$. Además, estas coincidencias corresponden a los momentos en que el Sol aparece alineado con el azimut de $187.6^{\circ}$.

\section{COMENTARIOS FINALES}

Una de las formas de expresión por medio de esculturas que reflejan la existencia de actos rituales y registros calendáricos, es la erección de Estelas ricamente decoradas con auge en el período Clásico Maya. En ellas, además de fechas de conmemoración o de registro de ciclos (Katunes), aparecen los gobernantes ataviados con ricas prendas y portando bastones decorados o rituales. En el período llamado Decadente de Dzibilchaltún, aparentemente, esta costumbre fue relegada y así, se pueden encontrar estelas lisas que bien pudieran haber sido trabajadas en un período previo y que pueden haber sido erigidas con un objetivo en mente.

Además, el poder político y/o religioso en una sociedad se hace evidente cuando existe en ella el registro del tiempo y por ende la prevalencia de un Calendario. En el caso Maya, el sofisticado sistema de cuenta de los días con la Cuenta Larga, el año Haab y el período Tzolkin hacen por demás evidente la existencia de una élite con sacerdotes-astrónomos o astrónomos-sacerdotes que ejercen cierto poder. En Dzibilchaltún, en la época denominada Decadente, algunos años antes de la conquista, la ciudad desarrolló una vocación ritual que se manifiesta en escasas nuevas construcciones. Entre ellas destaca el levantamiento de tres estructuras (Str.47, Str.48 y Str.49) con estelas, presuntamente originarias del período Temprano II, cuyas dimensiones y orientación sugieren un objetivo gnomónico. Su orientación refleja la búsqueda de la observación del Sol a $45^{\circ}$ en el Solsticio de Invierno con la sombra de las Estelas iguales a su altura; $y$, con las alturas de las mismas relacionadas unas con otras a través de sus sombras: suma de sombras de $\$ 4$ igual a $\$ 6$ y altura de $\$ 5$ igual a suma de las Sombras de S6.

Como la observación de sombras de un gnomon, o como en el presente caso, de una estela puede considerarse como una actividad geométrica pues en ella se ven involucrados elementos continuos, es lógico suponer que las dimensiones de dichas sombras traten de ser contadas (o mejor aún, relacionadas) por medio de unidades al elemento que las produce. Vemos aquí, entonces, con la relación entre las sombras de las estelas y sus alturas, una expresión concreta de los que John Stillwell aborda al considerar que las diferencias entre geometría y aritmé- 
tica son solo aparentes pues existe una relación profunda entre ellas, ejemplificado por el Teorema de Pitágoras [19].

Finalmente, podemos sugerir que las Estelas Calendáricas de Dzibilchaltún, aquí analizadas, constituirían un ejemplo de edificación con objetivos rituales y reflejo de un poder existente que como dice León Portilla, "los sabios mayas del horizonte clásico no fueron los primeros ni los únicos que consagraron su atención al tema del tiempo, [pero] si fueron los que con el más obsesionante interés desarrollaron ésta, que pudo haber sido herencia en común de la antigua cultura mesoamericana, hasta llegar a crear sistemas cronológicos con módulos y cómputos de una precisión que hoy nos parece inverosímil" [20].

\section{RECONOCIMIENTOS}

Deseo agradecer a la UNISON por el apoyo recibido para mi año sabático y a la UNAM, en especial al Instituto de Investigaciones Estéticas por haberme recibido en este período. Al Dr Jesús Galindo Trejo y al Dr Estanislao Ivanisciewski agradezco sus comentarios y orientaciones durante la preparación de este trabajo.

\section{BIBLIOGRAFÍA}

[1] J. Galindo Trejo, Arqueoastronomía en la América Antigua. Cd México: México: CONACYT, 1994.

[2]L. Fogelin, "The Archaeology of Religious Ritual", Annual Review of Anthropology, Vol. 36, pp. 55-71, 2007.

[3] M. Looper, Lighting Warrior: Maya art and kingship of Quirigua. Austin, USA:The University of Texas Press, 2003.

[4] D. Stuart, "Shining Stones: Observations on Ritual of Early Maya Stelae", in Place of Stone Monuments pp. 285-297. Ed. Julia Guernsey et al. Dumbarton Oaks Research Library and Collection, 2010.

[5] E. W. Andrews, "Lost City of the Maya". National Geographyc Magazine vol. 115, pp. 91 - 109, 1959.

[6] E. B. Kurjack, "Introduction to the Map of the Ruins of Dzibilchaltun, Yucatan, Mexico". Middle American Research Institute Publication Num. 47. EUA:Tulane University,1979.
[7] E. W. Andrews IV, E. W. Andrews V, "Excavations at Dzibilchaltun, Yucatan, Mexico". Middle American Research Institute Publication Num. 48. EUA:Tulane University, 1980.

[8] E.W. Andrews V, "Dzibilchaltún". Handbook of Middle American Indians. EUA: Ed. Bricker, V.R. \& J.A. Sabloff, supp. $1,1981$.

[9] S. T. Evans, Ancient Mexico and Central America: Archaeology and Culture History. London, UK: Thames \& Hudson, 2008.

[10] (2016) Google Earth, Accessed july 2016.

[11] E. M. Linton "From Eudoxus to Einstein: A history of Mathematics and Astronomy", Cambridge, UK: Cambridge Univ. Press 2004.

[12] R. Maldonado, A. Uriarte, G. Santiago, E. Acevedo, "Informe Técnico. Temporada 2001-2002". Proyecto Arqueológico Dzibilchaltún Num. 30.230. Cd. De México, México: INAH, 2003.

[13] R. Maldonado, "Proyecto Arqueológico Dzibilchaltún". Informe de Proyecto Num. 30-220. Cd. De México, México: INAH, 2003.

[14] C. Alvarez, Diccionario Etnolingüística del Idioma Mayayucateco colonial. I El Mundo Físico, Cd. De México: UNAM, 1980.

[15] A. Barrera Vásquez, Alfredo, Diccionario maya cordemex :Maya-español español-maya, Cd. De México, México: Cordemex, 1980.

[16] A. Villa Rojas, Apéndice de: Tiempo y realidad en el pensamiento maya. Cd. De México, México: Instituto de Investigaciones Históricas, UNAM, 1981.

[17] R. Perez-Enriquez, "Plato's Triangle and gnomonic factor: An application to Herodotus' Oracles", Mediterranean Archaeology and Archaeometry vol. 14 Num. 3, pp. 45 - 53, 2014. [Online] Available: http://maajournal.com/ Issues/2014/Vol14-3/Full5.pdf.

[18] E. Solis Alcalá, Diccionario Español-Maya. Mérida, Yucatán: Ed. YIKAL MAYATHAN, 1949.

[19] J. Stillwell, Mathematics and Its History. Springer-Verlag, 1989. [Online] Available: http://www.springer.com/us/ book/9781441960528.

[20] M. León Portilla Tiempo y realidad en el pensamiento maya, Cd. De México, México: UNAM, 1986.

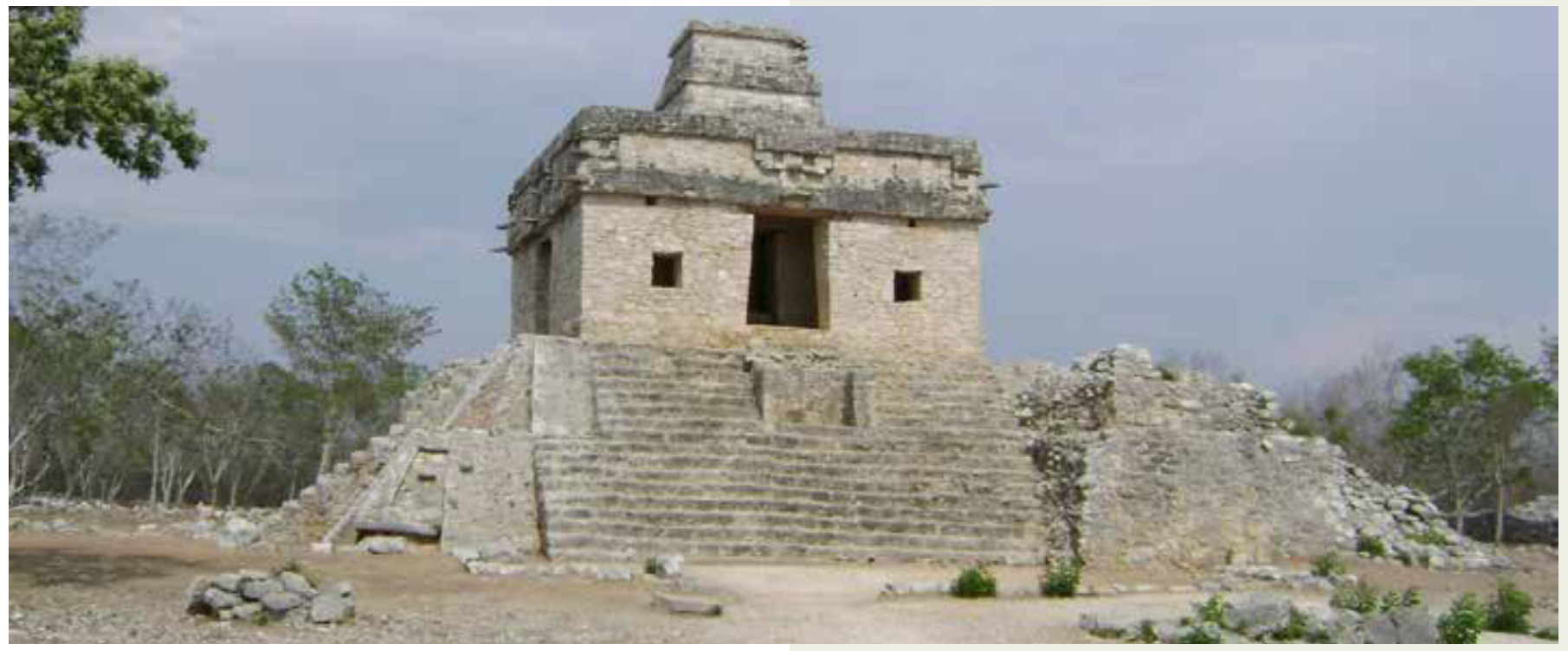

